want to start by thanking Editor-inChief Ron Ayling for providing an opportunity to write the guest editorial for this issue. I will use it to try to better inform our membership of some of the things the Institute is doing across the country to promote a positive and accurate message about forestry to the public, and also to encourage all members to do what they can as well, at every opportunity-i.e., walk the walk, not just talk the talk, as the axiom goes. These are just a few examples, and I could provide many more if space permitted.

Through annual teachers' forestry tours in different regions, often run by our members with many partners and sponsors, we have reached thousands of teachers directly over the past decade and tens of thousands of teachers and students indirectly. I am most closely connected to the teachers' tour at the Canadian Ecology Centre near Mattawa, Ontario (the location of our national office) and I can say with confidence that all participating teachers gain a totally new and positive perspective of forestry. And we accomplish this without propaganda or preaching-we simply show what we do and why, with honesty, professionalism and passion. We let them make up their own minds about forestry, but with better information and first-hand experience in the forest and with forest practitioners-a much more comprehensive presentation than the typical 30-second soundbite that many of us normally experience and use to help make our judgements and decisions about things. If you want to see and read for yourself, to get an idea of what we actually do, a Teachers' Forestry Tour video is available on CIFTube, our own YouTube channel, along with teacher testimonials with links at: http://cif-ifc.org/site/ teacherstour.

There is also plenty of general outreach to the public across our $18 \mathrm{Sec}$ tions, particularly during national forest week-with numerous successful events delivered every year. Some of our members are doing a lot when it comes to talking to the public about forestry.
Along with our partner-the Canadian Forestry Association, and as a result of a recent agreement with the Forest Products Association of Canada (FPAC), this year's National Forest Week theme will be The Greenest Workforce-aligning with FPAC's new recruitment campaign. This closing of ranks with our partners presents an exciting opportunity not only to promote a positive message about forestry, but also to encourage young people to consider related career paths that allow them to make a real difference in both urban and rural settings. National Forest Week (NFW) and National Tree Day have both been really gaining ground the past few years. A NFW activities video collage can be viewed at: http://www.youtube.com/ watch? $=\mathbf{j U}$ UNNdzI7VQ.

The Institute has not at all been quiet when it comes to speaking out and garnering successful uptake in the national news media as well, and here again are just a few examples. Our letter to Rona about misinformation in their television commercials, and an associated media release resulted in pick-up across Canada, and in particular British Columbia, including the Vancouver Sun. Rona changed the commercials and I like to think we helped, although other groups also went public at the same time with the same message. Our letter to TD Bank and a media advisory about their Forests Program also was well received when we suggested that their messaging be based more on sustainable use of forests. We also received extensive national and international coverage, including $\mathrm{CBC}$ radio, when former President Fred Pinto made the shortlist for the United Nations Forest Hero Award last year. These letters and media advisories and releases can all be viewed on our Web site at: http://cififc.org/site/news_releases.

In closing, I hope that all members are aware of these and many other Institute initiatives and outreach efforts-so many in fact that it is hard to keep track; but I also ask that everyone looks for opportunities to promote a positive message about forestry to everyone, everywhere. After all, we truly are
Canada's greenest workforce and the sense of pride we take in our work, and in our livelihoods, is what makes our story so compelling to students and teachers. You do not have to preachjust give them an alternate, accurate, professional and passionate storyline that better informs their values and perspectives. Our grassroots approach actually has a surprising amount of power and reach, and we need to remember and believe that each of us can make a difference. So when it comes to positive forestry messages, please walk the walk!

John Pineau

Chief Executive Officer Canadian Institute of Forestry

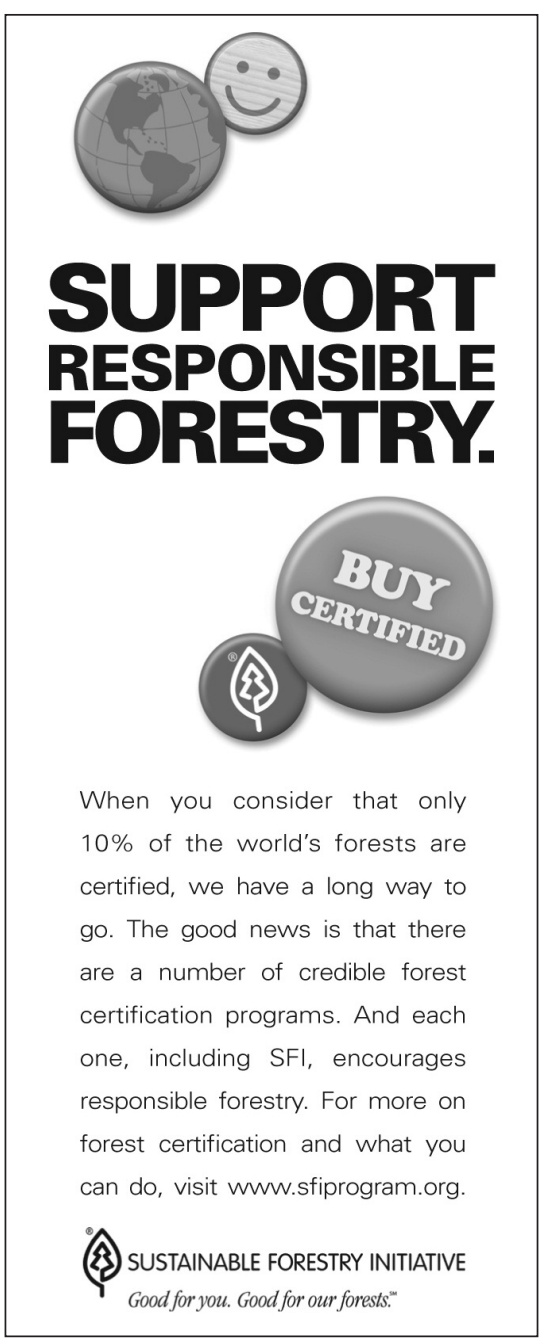




\section{De la parole au geste}

J tiens en premier lieu à remercier le rédacteur en chef Ron Ayling de mavoir offert la possibilité de rédiger léditorial de collaboration spéciale de ce numéro. Je vais en profiter pour tenter de mieux informer nos membres de ce que l'Institut fait dans l'ensemble du pays pour promouvoir une image positive et précise de la foresterie aux yeux du public et également pour encourager tous les membres à faire eux aussi ce qu'ils peuvent lorsqu'une occasion se présente-en sorte, passer de la parole au geste et ne pas parler pour parler comme on dit souvent. Il ne sagit que de quelques exemples et je pourrais en citer beaucoup plus si l'espace me le permettait.

Suite aux excursions techniques annuelles en foresterie destinées aux enseignants et organisées dans différentes régions et souvent pilotées par nos membres et plusieurs de nos partenaires et commanditaires, nous avons pu rejoindre directement des milliers d’enseignants au cours de la dernière décennie ainsi que des dizaines de milliers d'enseignants et d'étudiants indirectement. Je suis plus étroitement impliqué dans l'excursion pour les enseignants organisée au Centre écologique du Canada situé près de Mattawa en Ontario (le site de notre bureau national) et je peux affirmer avec certitude que tous les enseignants qui y participent acquièrent une perspective totalement nouvelle et positive de la foresterie. Et nous avons atteint ce résultat sans faire de propagande ni de prêche auprès des enseignants-nous leur avons simplement exposé ce que nous faisons et pourquoi nous le faisons, en toute honnêteté, professionnalisme et ferveur. Nous les avons laissés réfléchir sur ce qu'est la foresterie, mais avec de meilleures informations et un apprentissage technique en forêt en compagnie d'intervenants en foresterie -un exposé beaucoup plus complet que les extraits sonores de 30 secondes dont nous avons l'habitude et qui sont utilisés pour éclairer notre jugement et nous aider à prendre des décisions. Si vous désirez voir et consulter le programme de ces activités pour vous faire une idée de ce que nous faisons concrètement, vous pouvez consulter une vidéo sur l'excursion technique en foresterie pour les enseignants sur IFCTube, notre propre chaîne YouTube, ainsi que les témoignages des enseignants au : http://cif-ifc.org/site/teacherstour.

Nos 18 sections mettent sur pied plusieurs activités de sensibilisation du grand public, notamment lors de la Semaine nationale de larbre et des forêts-plusieurs d'entre elles répétées chaque année. Plusieurs de nos membres font les choses en grand lorsqu'il est question de s'adresser au grand public et de parler de foresterie. En collaboration avec notre partenairel'Association forestière canadienne et suite à une entente récente avec l'Association des produits forestiers du Canada (FPAC), le thème de la Semaine nationale de l'arbre et des forêts de cette année sera-La main d'œuvre la plus verte-aligné sur la nouvelle campagne de recrutement de FPAC. Ce resserrement des rangs parmi nos partenaires représente une occasion formidable non seulement de promouvoir un message positif au sujet de la foresterie, mais aussi d'encourager les jeunes à envisager un cheminement de carrière qui leur permet d'apporter une contribution significative tant en milieu urbain que rural. La Semaine nationale de l'arbre et des forêts (SNAF) et la Journée nationale de l'arbre sont toutes deux des activités qui ont pris de l'ampleur au cours des dernières années. Une vidéo constituée des activités de la SNAF peut être consultée à : http://www.youtube.com/ watch?v=jUyNNdzI7VQ.

L'Institut a été loin d'être effacé lorsqu'il est à propos de s'exprimer et d'obtenir l'attention des médias nationaux et en voici quelques exemples. Notre lettre adressée à Rona au sujet de l'information erronée contenue dans leurs annonces publicitaires présentées à la télévision, ainsi que le communiqué de presse qui a suivi, ont été repris dans l'ensemble du Canada et en particulier en Colombie-Britannique et notamment par le Vancouver Sun. Rona a modifié ses commerciaux et je voudrais croire que nous avons eu une influence, malgré que d'autres groupes aient également occupé l'espace public pour souligner la même erreur. Notre lettre acheminée à la Banque TD et un avis aux médias concernant leur programme de foresterie ont été accueillis favorablement alors que nous leur proposions que leur message porte un peu plus sur l'utilisation durable des forêts. Nous avons également fait la manchette sur la scène nationale et internationale lorsque l'ancien président Fred Pinto a été désigné parmi les finalistes du Prix Héro de la Forêt décerné par les Nations Unies l'an dernier. Ces lettres, les avis aux médias et les communiqués peuvent être consultés sur notre site internet à http://cif-ifc.org/site/news_releases.

En terminant, jespère que tous les membres prendront connaissance de ces réalisations et des nombreuses activités de l'Institut ainsi que de ses initiatives de sensibilisation-tellement nombreuses qu'il est difficile de toutes les suivre; mais je demande à tous et chacun d'identifier les opportunités de transmettre un message positif sur la foresterie à toutes les personnes de votre entourage. Vous n'avez pas besoin de prêcher-mais de seulement présenter une version différente, précise, professionnelle et empreinte de passion qui touche adéquatement leurs valeurs et leurs points de vue. Notre approche locale démontre dans les faits un haut niveau de puissance et de portée et nous devons nous rappeler et considérer que chacun d'entre nous peut faire la différence. Alors, lorsqu'il est question d'un message positif sur la foresterie, s'il vous plait, passez de la parole au geste !

John Pineau

Président directeur général Institut forestier du Canada 